\title{
LeGes
}

Seraina Herzberg / Stefan Rieder

\section{Das Verhältnis von KMU und zivilrechtlicher Justiz}

\section{Beobachtungen zu Vollzug und Wirksamkeit}

Gerät ein Unternehmen mit einem Kunden in Streit und können sich die Parteien nicht einigen, gehen sie vor Gericht. So würde man vermuten. Dem ist in der Praxis aber nicht so: Namentlich kleine und mittlere Unternehmen treten selten den Gang vor ein Zivilgericht an. Der Beitrag geht diesem möglichen Vollzugsdefizit nach und erörtert zwei Gruppen von Gründen: Die erste Gruppe geht von den Ressourcen und dem Selbstverständnis der Unternehmen aus. Die zweite Gruppe von Gründen ergibt sich aus der Ausgestaltung der Verfahren und dem Selbstverständnis der Gerichte. Abschliessend wird über die Notwendigkeit einer Veränderung des Status quo diskutiert.

Beitragsart: Wissenschaftliche Beiträge

Zitiervorschlag: Seraina Herzberg / Stefan Rieder, Das Verhältnis von KMU und zivilrechtlicher Justiz, in: LeGes 31 (2020) 1 


\section{Inhaltsübersicht}

1. Einleitung

2. Perspektive der KMU: Theoretische und empirische Befunde zu Vollzug und Wirksamkeit der Streitschlichtung bei KMU im Zivilrecht

2.1. Theoretische Überlegungen

2.2. Empirische Befunde zum Verhältnis Justiz und Unternehmen

2.3. Zwischenfazit: Endogene Faktoren und fehlende Ressourcen verhindern die Anwendung zivilprozessualer Streitschlichtungsinstrumente

3. Perspektive des Rechts: Justiznahe Gründe zu fehlendem Vollzug und fehlender Wirksamkeit der Streitschlichtung bei KMU durch die Justiz

3.1. Prohibitiv hohe (Prozess-)Kosten

3.2. Justiz enttäuscht Gerechtigkeitserwartungen

3.3. Informationsdefizite der KMU sind hoch

3.4. Zwischenfazit: Hohe Kosten, enttäuschte Erwartungen und fehlende Informationen hemmen die Anwendung und Wirksamkeit zivilprozessualer Streitschlichtungsinstrumente

4. Schlussfolgerungen

4.1. Endogene und exogene Gründe für die geringe Nutzung von Zivilgerichten durch KMU

4.2. Veränderungsbedarf: Pro und Contra

\section{Einleitung}

[1] Es ist eine gerichtsnotorische Tatsache, dass Unternehmen die Justiz wenig in Anspruch nehmen. Umso mehr erstaunt, dass wissenschaftliche Untersuchungen in der Schweiz weitgehend fehlen, die den Gründen für diese Feststellung nachgehen würden. Einzig der Volksmund artikuliert ein gewisses generelles Unbehagen gegenüber der Justiz mit Aussagen wie «Man kann auch recht behalten, ohne es zu haben» oder «Vor Gericht bist du in Gottes Hand».

[2] Der Beitrag möchte diesem Unbehagen und dem Verhältnis zwischen Unternehmen und der Justiz (den Gerichten) am Beispiel der KMU nachgehen. Allgemein geht es um die Frage, warum KMU in zivilrechtlichen Konflikten selten Gerichte anrufen und welche Gründe für die entsprechenden Vollzugsdefizite (RIEDER et al. 2014, S. 576-577) und die fehlende Wirksamkeit verantwortlich sind. Die allgemeine Fragestellung lässt sich mittels folgender drei Teilfragen konkretisieren:

- Welche Daten liegen zur Beschreibung des Vollzugs und der Wirksamkeit der Streitschlichtung via Zivilgerichte vor?

- Welche Gründe lassen sich aus Sicht der KMU finden, die eine beobachtete geringe Inanspruchnahme der Zivilgerichte zu erklären vermögen?

- Welche Gründe gibt es seitens der Gerichte, die die Anwendung und Wirksamkeit der Streitschlichtung durch die Zivilgerichte erschweren?

[3] Um den Gegenstand einzugrenzen, konzentriert sich der Beitrag innerhalb der KMU auf sogenannte Mikrounternehmen. ${ }^{1}$ Mikrounternehmen unterscheiden sich in vielerlei Hinsicht von

1 Das Bundesamt für Statistik definiert KMU als Unternehmen mit einer Beschäftigungszahl von 0 bis 249 Vollzeitstellen. $99 \%$ aller marktwirtschaftlichen Betriebe in der Schweiz gehören damit der Gruppe der KMU an (STATENT des BFS aus dem Jahr 2017); Mikrounternehmen sind gemäss Bundesamt für Statistik Unternehmen 
sogenannten Klein- und Mittelunternehmen: Beispielhaft seien hier die Wachstumsresistenz, eingeschränkte finanzielle und personelle Mittel sowie die ausgesprochene Exposition im wirtschaftlichen Umfeld genannt (m.w.H. Kaufmann et al. 2010, S. 4 ff.). Dennoch haben Mikrounternehmen eine hohe wirtschaftliche Bedeutung, stellen sie doch nicht weniger als rund 40 Prozent der Beschäftigten in KMU und gesamthaft rund einen Viertel aller Beschäftigten. Wirtschaftspolitik und Forschungstätigkeit richten sich allerdings auf die grösseren Klein- und Mittelunternehmen aus. Die «eigentümliche Geschäfts- und Konfliktkultur» von Mikrounternehmen ist hingegen wenig erforscht (Kaufmann et al. 2010, S. 6). Sie könnte aber schon nur aufgrund ihrer quantitativen Bedeutung einen bedeutenden Einfluss auf das Verhältnis zwischen KMU und Justiz haben, weshalb wir sie hier ins Zentrum stellen. Gleichzeitig fokussieren wir uns auf Zivilprozesse auf kantonaler Ebene.

[4] Der Arbeit liegt einerseits eine Dokumentenauswertung zugrunde: Ausgewertet wurden Literatur- und Medienberichte, Ergebnisse aus publizierten KMU-Befragungen sowie Gerichtsstatistiken. Andererseits wurden die Botschaft zur Zivilprozessordnung (ZPO) sowie die Materialien rund um die laufende Revision der Zivilprozessordnung herangezogen. Ergänzend wurden explorative Interviews bei Anwälten und Richtern (zwei Anwälte, zwei Richter) durchgeführt. Schliesslich wurden die Ergebnisse an einem interdisziplinären Seminar der Universität präsentiert und diskutiert. Der Beitrag versteht sich in der Folge nicht als umfassende Analyse, sondern als erste Annäherung an das Thema.

[5] Der Beitrag ist wie folgt aufgebaut: Zunächst nehmen wir eine Analyse aus Sicht der KMU vor. Dabei wird geprüft, welche theoretischen und empirischen Gründe dafür verantwortlich sind, dass KMU in Zivilstreitigkeiten selten die Justiz in Anspruch nehmen und diese somit kaum eine Wirksamkeit entfalten kann. Anschliessend wechseln wir die Perspektive und stellen die Frage, ob und inwiefern es Gründe auf Seiten der Justiz gibt, die KMU vom Gang an ein Zivilgericht abhalten und somit zu einem Vollzugsdefizit führen. Der Beitrag endet mit einer Diskussion und einem Ausblick.

\section{Perspektive der KMU: Theoretische und empirische Befunde zu Vollzug und Wirksamkeit der Streitschlichtung bei KMU im Zivilrecht}

[6] Wir beginnen unsere Betrachtung aus Sicht der Perspektive der KMU. Aus einer theoretischen Sichtweise ist die neue Institutionenökonomik hilfreich, um das Verhältnis zwischen Gerichten und KMU zu beschreiben. Anschliessend stellen wir die theoretischen Überlegungen den vorhandenen empirischen Beobachtungen gegenüber und ziehen am Schluss des Kapitels ein erstes Fazit.

\subsection{Theoretische Überlegungen}

[7] Unternehmen stehen verschiedene Institutionen zur Verfügung, um Konflikte zu lösen. Die staatliche Justiz ist nur eine dieser Institutionen. Wird die staatliche Justiz nicht zur Konfliktlösung herangezogen, liegt es nahe, nach anderen Institutionen zu suchen, die an die Stelle der Jus-

mit bis zu 9 Vollzeitangestellten. Innerhalb der KMU sind dies $90 \%$ der Betriebe, die knapp $40 \%$ der Arbeitsplätze anbieten. 
tiz treten. Es werden nachfolgend einige Regelüberwachungssysteme (Institutionen) dargestellt, welche für die Konfliktlösung im Umfeld von Unternehmen zum Einsatz kommen können. Dazu bedienen wir uns eines Modells aus der Neuen Institutionenökonomik. Diese geht davon aus, dass Wachstum und Entwicklung eines Landes entscheidend von den jeweils gültigen Institutionen abhängt (Von Escher 2008, S. 64). Der Institutionenbegriff wird weit gefasst: Institutionen sind formelle und informelle gesellschaftliche (Spiel-)Regeln einschliesslich der Mechanismen ihrer Durchsetzung. Der Begriff Institutionen beinhaltet also eine Regel- wie auch eine Sanktionskomponente (Voigt 2009, S. 26 ff.).

[8] Grundsätzlich wird zwischen internen und externen Institutionen unterschieden. Interne Institutionen sind Regeln, deren Durchsetzung nicht unter Rückgriff auf den Staat erfolgt. Ein Verstoss gegen solche Regeln wird innerhalb des jeweiligen Subsystems der Gesellschaft sanktioniert. Externe Institutionen sind im Gegensatz dazu Regeln des gesetzten positiven Rechts, deren Durchsetzung unter Rückgriff auf den Staat erfolgt. Regelverstösse gegen solche externe Institutionen werden staatlich sanktioniert (VoIgt 2009, S. 31). Tabelle 1 gibt einen Überblick über die verschiedenen Institutionentypen und ihre Eigenschaften.

Tabelle 1: Institutionentypen im Kontext von KMU

\begin{tabular}{|l|l|l|l|}
\hline $\begin{array}{l}\text { Institutionen- } \\
\text { typ }\end{array}$ & Regel & Überwachung & Beispiel \\
\hline Intern Typ 1 & Konvention & Selbstüberwachung & $\begin{array}{l}\text { Grammatikalische } \\
\text { Regeln der Sprache }\end{array}$ \\
\hline Intern Typ 2 & Ethische Regel & Imperative Selbstbindung & $\begin{array}{l}\text { Dekalog, Kategorischer } \\
\text { Imperativ }\end{array}$ \\
\hline Intern Typ 3 & Sitte & $\begin{array}{l}\text { Spontane Überwachung } \\
\text { durch andere Akteure }\end{array}$ & $\begin{array}{l}\text { Gesellschaftliche } \\
\text { Umgangsformen }\end{array}$ \\
\hline Intern Typ 4 & $\begin{array}{l}\text { Formelle private } \\
\text { Regel }\end{array}$ & $\begin{array}{l}\text { Geplante Überwachung } \\
\text { durch andere Akteure, } \\
\text { Schiedsgerichte }\end{array}$ & $\begin{array}{l}\text { Selbstgeschaffenes } \\
\text { Recht der Wirtschaft }\end{array}$ \\
\hline Extern & $\begin{array}{l}\text { Regel positiven } \\
\text { Rechts }\end{array}$ & $\begin{array}{l}\text { Organisierte staatliche } \\
\text { Überwachung, Justiz }\end{array}$ & Privat- und Strafrecht \\
\hline
\end{tabular}

Quelle: KIWIT/VOIGT 1997, S. 124, leicht modifizierte Darstellung

[9] Die Justiz wird in diesem Modell als externe Institution verstanden, die Konfliktlösungsinstrumente im Rahmen des Zivilrechts anbietet (Klagen, Prozesse, Rekursmöglichkeiten usw.), die von KMU zur Streitschlichtung mit ihren Kundinnen und Kunden angewendet werden können (wenn etwa Zahlungen ausbleiben oder Uneinigkeit über die ordnungsgemässe Erfüllung eines Vertrags besteht).

[10] Folgt man nun der oben dargestellten Tabelle, wird sogleich klar, dass die externe Institution des Rechts keineswegs die einzige Institution zur Konfliktlösung darstellt. Vielmehr verfügen die Unternehmen ihrerseits über interne Institutionen, die sie bei Konflikten heranziehen: Es können dies beispielsweise ethische Regeln und Sitten (Institutionentyp 3) sein, deren «Überwachung» durch die imperative Selbstbindung respektive die spontane Überwachung durch andere Akteure (z. B andere KMU) erfolgt. Die Justiz kommt in solchen Fällen nicht zum Zug, die gesetzlich vorgesehene Streitschlichtung wird gar nicht angewandt und entfaltet somit auch keine Wirkung. 
Man könnte von einem Substitutionseffekt sprechen, insofern die internen Institutionentypen 14 die externen Institutionentypen ersetzen. Mit nachfolgendem Zitat lässt sich dies an Hand des Institutionentyps 3 illustrieren. Dabei wird ein Handwerker bei Kaufmann et al. (2010, S. 75/102) wie folgt zitiert:

«Ich liefere nur gute Qualität, dann habe ich keine Probleme! Sollte etwas nicht stimmen, komme ich selbstverständlich entgegen.»

[11] Hier bindet sich das Kleinunternehmen an interne Institutionen: Der Berufsstolz des Inhabers und seine Orientierung an ethischen Regeln und Sitten sind wichtiger als geschriebene Rechte und Pflichten. Die Überwachung dieser Regeln erfolgt dabei durch Selbstbindung, durch die Kundschaft oder durch andere Unternehmen, die schlechte Qualität publik machen würden. [12] Aus dem theoretischen Modell lässt sich eine erste Antwort auf unsere Ausgangsfrage ableiten, weshalb die Justiz von den KMU kaum in Anspruch genommen wird und somit wenig Wirkungen entfalten kann: Wenn sich Unternehmen primär an internen Institutionen orientieren, ergibt sich daraus ein distanziertes Verhältnis zwischen Justiz und Unternehmen. Die relevanten Variablen, die dieses Verhältnis beeinflussen, wären auf Seiten der Unternehmen deren internalisierte ethische Regeln, Sitten, Gewohnheiten und formelle selbstgeschaffene Regeln. Aus theoretischer Sicht ist es somit nachvollziehbar, dass die internen Institutionen Konflikte befriedigend zu lösen vermögen und es daher nur in Ausnahmen dazu kommt, dass externe Instanzen (Gerichte) für eine Konfliktlösung angerufen werden. Vor allem bei Mikrounternehmen scheint dieser Zusammenhang plausibel, da meist der Inhaber oder die Inhaberin auf Basis der für ihn oder sie gültigen Sitten, Konventionen und ethischen Standards die Arbeitsweise im Unternehmen dominiert. Ferner ist es sinnvoll anzunehmen, dass bei Mikrounternehmen, die in einem begrenzten geografischen Umfeld tätig sind, eine Selbstkontrolle oder spontane Überwachung durch Dritte (Kundschaft und Konkurrenz) stattfinden dürfte. Die zivilprozessual vorgesehenen Streitschlichtungsinstrumente werden somit durch interne Institutionen in den Hintergrund gedrängt und kommen gar nicht zum Einsatz. Faktisch findet in der Folge kein oder nur ein sehr geringer Vollzug gerichtlicher Streitschlichtung statt.

\subsection{Empirische Befunde zum Verhältnis Justiz und Unternehmen}

[13] Wie weit lassen sich die auf Basis der theoretischen Überlegungen erwartete geringe Anrufung der Zivilgerichte, der geringe Vollzug und die geringe Wirksamkeit gerichtlicher Streiterledigung bei KMU und Mikrounternehmen mit empirischen Daten untermauern?

[14] Wie bereits eingangs erwähnt, nutzen juristische Personen das Gericht signifikant wenig, um Konflikte zu lösen. Dieser allgemeine Befund wird von den meisten Gerichten und Expertinnen und Experten geteilt. Sucht man aber allgemein zugängliche statistische Daten in Bezug auf KMU und deren Zugang zu kantonalen Gerichten, so tut man sich schwer: Es existieren in der Schweiz bis dato keine einheitlichen Vorgaben für die Erfassung von statistischen Daten in Bezug auf zivilrechtliche Verfahren. ${ }^{2}$ Die veröffentlichten Daten sind aufgrund der unterschiedlichen

2 Mit der laufenden Revision der ZPO ist ein neuer Art. 401a ZPO zu «Statistik und Geschäftszahlen» vorgesehen, der erstmals eine gesamtschweizerische gesetzliche Grundlage für statistische Erhebungen in Zivilprozessen schaf- 
Gerichtsorganisation in den Kantonen und weiterer kantonaler Besonderheiten (trotz vereinheitlichter ZPO) nur bedingt vergleichbar. So wird etwa im Kanton Zürich bei den Falleingängen an den Gerichten nicht erfasst, ob es sich um eine natürliche oder eine juristische Person handelt, da dies für den Rechenschaftsbericht nicht relevant ist. Somit bleiben nur indirekte Abschätzungen via Aussagen aus den geführten explorativen Interviews oder der Rückgriff auf bestehende Untersuchungen, die wir im Folgenden kurz ausführen.

[15] Kaufmann et al. (2010) haben in einer qualitativen Befragung von KMU und Expertinnen und Experten sowie in einer schriftlichen Befragung von KMU verschiedene empirische Untersuchungen zum Thema KMU und Konflikte durchgeführt. Die Ergebnisse zeigen, dass Mikrounternehmen unter einem starken Konkurrenzdruck stehen. Dies führt einerseits zu Innovationen, um sich im Markt behaupten zu können. Andererseits besteht ein hoher Preisdruck (Kaufmann et al. 2010, S. 11). In dieser Situation bieten sich für Mikrounternehmen primär folgende Institutionen zur Lösung von Problemen mit Kundinnen und Konkurrenz an: Das Austragen von Konflikten, der Vertrag und die Kooperation. Die Austragung eines Konflikts führt in der Regel zu Kontakten mit der Justiz. Dass diese Kontakte zur Justiz dennoch selten stattfinden, ist auf die Kulanz oder das Nachgeben der Mikrounternehmen zurückzuführen. Dies erfolgt meist unter Rückgriff auf persönliche Kompetenz und Haltung des einzelnen Unternehmers oder der Unternehmerin (Kaufmann et al., 2010, S. 100). Die empirische Beobachtung von Kaufmann et al. scheint somit den vorne theoretisch postulierten Substitutionseffekt von zivilprozessualen Streitschlichtungsinstrumenten durch interne Institutionen der KMU zu bestätigen.

[16] Weiter haben die Untersuchungen gezeigt, dass KMU-Geschäftsbeziehungen stark auf Vertrauen und weniger auf schriftlichen Verträgen aufbauen. Eine Zusammenarbeit wird als gut bewertet, wenn Parteien sich darauf verlassen können, dass bei Problemen informelle Lösungen gefunden werden. Es gelten primär kulturell verankerte Grundsätze wie Treu und Glauben als Spielregeln (Kaufmann et al. 2010, S. 56). In Bezug auf Kooperation hat der Mikrounternehmer wenig Spielraum; er muss weiter kooperieren, auch wenn Schwierigkeiten auftauchen: Je älter und vorteilhafter die Kooperation, desto grösser ist das Entgegenkommen bei Konflikten. Dabei spielen asymmetrische Kräfteverhältnisse eine wichtige Rolle (Kaufmann et al. 2010, S. 44 f.). Die Kooperation ist in der Folge oft von der Kundin, vom Kunden oder von der Konkurrenz aufgezwungen und letztendlich die einzige Möglichkeit, im Konkurrenzdruck zu bestehen. Weder bestehe eine geeignete Organisation noch das notwendige Wissen, um einen Konflikt vor Gericht zu bewältigen (KaUfManN et al. 2010, S. 10).

[17] Allerdings lässt sich feststellen, dass mit zunehmender Grösse des Betriebs externe institutionelle Angebote (zum Beispiel Gerichte) stärker genutzt werden. Umgekehrt wird konstatiert, dass je kleiner der geografische Aktionsradius von KMU ist, desto stärker die Wirkung gesellschaftlicher Ordnungsfunktionen und desto weniger wichtig das Ordnungsinstrument Recht (KaufmanN et al., 2010, S.101 f.; von Escher 2008, S. 52). Kaufmann et al. schliessen aus der untersuchten Geschäfts- und Konfliktkultur, dass ein Mikrounternehmen nur in seltenen Ausnahmefällen den Schritt in ein Gerichtsverfahren wagen wird. Dies im Unterschied zum typischen Mittelunternehmen, das den Gang vor Gericht als Option im Gesamtkonzept der Geschäftstätigkeit sieht (Kaufmann et al., 2010, S. 129). Auch das Schlichtungsverfahren, das einfach, kostengünstig, niederschwellig und mit der Suche nach Vergleichen und Kompromissen auf den ersten Blick

fen soll. In der Vernehmlassung war der neue Artikel umstritten, insbesondere wurden Kostengründe und praktische Schwierigkeiten dagegen angeführt, vgl. Ergebnisbericht Rev. ZPO, S. 58. 
attraktiv für KMU wirkt, scheint die Dominanz der internen Institutionen nicht durchbrechen zu können (KaUfMANN et al., 2010 S. 123 f.).

[18] Wie weit lassen sich diese qualitativen Befunde quantitativ stützen? Mittels offizieller Statistiken ist dies wie oben geschildert nicht möglich. Hingegen lassen sich Schätzungen von Expertinnen und Experten heranziehen, die sich notgedrungen auf bestimmte Regionen oder Gerichte beschränken. So hat ein von uns befragter Bezirksrichter das Gesamtvolumen der Prozesse an Zürcher Bezirksgerichten, die KMU betreffen, auf 5 Prozent geschätzt. Bei den vereinfachten Verfahren ${ }^{3}$ beträgt der geschätzte Anteil immerhin ein Drittel. Dabei handelt es sich gemäss den von uns Befragten aber häufig um aussichtslose Forderungsprozesse, bei denen die beklagte Seite bereits zahlungsunfähig sei. Eine Konfliktschlichtung, die beiden Parteien etwas einbringen würde, liege daher nicht vor. Zudem halten die von uns befragten Richter wie auch die befragte Anwaltschaft fest, dass die geltenden formellen Vorgaben für eine Klage in der ZPO so hoch seien, dass KMU selten in der Lage seien, diese zu erfüllen. Die formalen Anforderungen der Verfahren machen somit ihre Nutzung schwierig: Die KMU rufen die Justiz aufgrund fehlender Ressourcen und Kompetenz nicht an. Es entsteht ein faktisches Vollzugsdefizit (RIeder et al. 2014; S. 576), das verhindert, dass die zivilprozessual vorgesehenen Verfahren eine Wirkung für KMU zu entfalten vermögen. Ein über mehrere Jahrzehnte tätiger Richter ergänzt dazu, dass die Zahl der KMU, die ein Gericht anrufen würden, schon immer bemerkenswert tief gewesen sei. Es kann in der Folge davon ausgegangen werden, dass KMU und insbesondere Mikrounternehmen wenige Berührungspunkte mit der Justiz aufweisen.

\subsection{Zwischenfazit: Endogene Faktoren und fehlende Ressourcen verhin- dern die Anwendung zivilprozessualer Streitschlichtungsinstrumente}

[19] Insgesamt lassen sich aus den theoretischen Überlegungen sowie den vorliegenden empirischen Ergebnissen aus Sicht der KMU drei Punkte festhalten.

- Die (spärlichen) quantitativen Daten und Schätzungen stützen die Beobachtung, dass KMU und Mikrounternehmen in Zivilstreitigkeiten vergleichsweise selten Gerichte anrufen. Es findet somit eine nur geringe oder gar keine Umsetzung von zivilprozessual vorgesehener gerichtlicher Streitschlichtung bei KMU und Mikrounternehmen statt. Dort, wo die Gerichte angerufen werden, scheinen die Forderungen oft so aussichtslos, dass kaum Wirkungen entstehen.

- Der Verzicht auf die Anrufung der Zivilgerichte durch die KMU lässt sich offenbar mindestens teilweise mit dem Vertrauen in interne und externe Institutionen erklären: Aufgrund ihrer Geschäfts- und Konfliktkultur bemühen Mikrounternehmen selten die Justiz. Sie bevorzugen die Abstützung auf interne Institutionen wie ethische Regeln, Sitte oder Gewohnheit, um Konflikte zu vermeiden oder zu lösen. Mit anderen Worten, das Vertrauen in interne Institutionen ist weit höher, als jenes in die externe Institution der Zivilgerichte.

3 Das vereinfachte Verfahren stellt geringere Anforderungen an die Parteien und weist einen vereinfachten Ablauf auf. Es ist als Verfahren für sozialsensible Bereiche wie Arbeits- oder Mietrecht, aber auch für Forderungsstreitigkeiten bis zu 30000 Franken konzipiert. 
- Schliesslich stehen den KMU oftmals nicht die notwendigen Ressourcen und das notwendige Knowhow zur Verfügung, um die formellen Anforderungen an eine Klage zu erfüllen. Insofern liegt ein faktisches Vollzugsdefizit vor.

[20] Zugespitzt könnte von einem «Nicht-Verhältnis» zwischen KMU und Zivilgerichten gesprochen werden: Die KMU, insbesondere Mikrounternehmen, und die Zivilgerichte bilden zwei unterschiedliche Systeme, die gegenseitig nicht kompatible interne und externe Institutionen aufweisen. ${ }^{4}$ Trifft dies zu, so vermag die fehlende Anwendung und Wirksamkeit der Justiz in unserem Kontext nicht zu erstaunen.

\section{Perspektive des Rechts: Justiznahe Gründe zu fehlendem Vollzug und fehlender Wirksamkeit der Streitschlichtung bei KMU durch die Justiz}

[21] Wenn KMU die Konfliktregelung ausserhalb der Justiz abwickeln, könnte dies auch mit Faktoren zusammenhängen, die auf Seiten der Justiz zu suchen sind. In der juristischen Lehre werden vor allem die hohen Kosten problematisiert, die KMU vom Gang an die Gerichte abhalten würden. Allerdings können auch nicht erfüllte Gerechtigkeitserwartungen sowie die Frage des Informationszugangs eine Rolle spielen. Wir gehen diesen drei Aspekten im Folgenden nach und prüfen, ob und inwiefern sie für den fehlenden Vollzug und die fehlende Wirksamkeit der Streitschlichtung im Zivilprozess bei KMU verantwortlich sind.

\subsection{Prohibitiv hohe (Prozess-)Kosten}

[22] Vor Einführung der ZPO existierten kantonale Prozessrechte mit unterschiedlich geregelten Prozesskosten (vgl. die Zusammenstellung bei StäHelın 2017a, S. 19 ff.). Die aktuelle Regelung der ZPO, die schweizweit seit dem 1. Januar 2011 gilt, beinhaltet zwei Ansätze bezüglich der Kosten:

- Allgemeine Vorschusspflicht gem. den Art. 98 und 102 ZPO: Es muss ein Kostenvorschuss in der Höhe der mutmasslichen Gerichtskosten als Eintretensvoraussetzung geleistet werden. De iure handelt es sich um eine Kann-Formulierung. De facto handelt es sich um eine MussRegelung: In allen Kantonen werden Vorschüsse gefordert (MEIER/SCHINDLER 2015, S. 76 ff.; etwas resigniert Zotsang 2015, S. 258; zum eigtl. vorgesehenen Ermessensspielraum vgl. Botschaft ZPO, S. 7293).

- Liquidationsregelung gem. Art. 111 ZPO: Der Prozessgewinner erhält den geleisteten Kostenvorschuss nicht vom Staat zurück, sondern muss diesen selber bei der Gegenseite einholen. Das Inkassorisiko wird vom Staat auf die Parteien überwälzt.

4 Zur Frage der «Kommunikation» zwischen unterschiedlichen Systemen s. LuHManN, S. 60 ff.; zu Verhältnis/ Interdependenzen zwischen internen und externen Institutionen siehe VoIGT, S. 32 f. / S. 64 ff., der von einer konfligierenden, einer komplementären oder einer substitutiven Beziehung zwischen internen und externen Institutionen spricht. 
[23] Diese neue Regelung wurde aus finanziellen Gründen auf Betreiben der Kantone eingeführt ${ }^{5}$ und führte zu direkten und indirekten Auswirkungen.

- In vielen Kantonen sind die Hürden, vor Gericht zu gehen, seit Einführung der ZPO höher geworden, und zwar als direkte Folge der neuen Kostenregelung. So sind beispielsweise im Kanton Zürich (er kannte nach altem Recht keine Kostenvorschusspflicht) die Eingänge an den Gerichten um knapp 20 Prozent zurückgegangen (vgl. Meier/Schindler 2015, S. 77 f.; Marti 2017, Rz. 28 f.; vgl. auch die Erhebung von Schmid 2017, S. 12 f.).

- Die befragten Anwältinnen und Anwälte raten KMU von einem Gang vor ein Zivilgericht ab, wenn der Streitwert unter 20000 Franken oder über 1 Million Franken liegt. Unter einem gewissen Streitwert können die gesamten Prozesskosten mit den Anwaltskosten nämlich höher als der Streitwert sein. Ferner steigen die Prozesskosten überproportional bei hohen Streitwerten, weil die Einnahmen aus diesen Prozessen offenbar von den Gerichten zur Quersubventionierung anderer Prozesse verwendet werden.

- Die Liquidität lässt es bei kleinen KMU oftmals nicht zu, die Prozesskosten vorzufinanzieren. Ferner können bei einer Prozessniederlage die Folgekosten (Prozesskosten, Anwaltskosten der Gegenpartei) ein KMU und insbesondere ein Mikrounternehmen existenziell gefährden. Entsprechend selten ist der Gang vor Gericht. In der Lehre wird die genannte Kostenregelung denn auch als mögliche Rechtswegsperre kritisiert. ${ }^{6}$

[24] Die Kritik hat auch medial Beachtung gefunden ${ }^{7}$, wobei von Seiten der Wirtschaftsverbände keine eigentliche Problematisierung im Sinne eines Agenda-Settings erkennbar ist. Eine Revision der ZPO unter dem Namen «Verbesserung der Praxistauglichkeit und Rechtsdurchsetzung» ist hängig. Diese strebt auch eine Änderung der Prozesskostenregelung an - der Erfolg ist aber ungewiss. $^{8}$

[25] Neben den geschilderten direkt wahrnehmbaren Auswirkungen der ZPO können die neuen Regelungen auch indirekte nicht intendierte Folgen nach sich ziehen. Hierzu berichtete ein interviewter Anwalt Folgendes: Gefragt, welche Strategien KMU denn verfolgen würden, um ihre Konflikte aussergerichtlich zu regeln, nannte er die Abschreibung der Beträge, die Einigung, eigene Vergleichsgespräche oder Vergleichsgespräche unter Beizug eines Anwalts. Bei Letzteren

5 Der Vorentwurf der ZPO sah als Kompromiss einen höchstens hälftigen Vorschuss (Art. 87 VE ZPO) sowie die Rückerstattung an die Parteien vor, soweit ihnen der Entscheid keine Kosten auferlegte (Art. 101 VE ZPO). In der Vernehmlassung löste dieser Vorschlag auf Seiten der Kantone grossen Widerstand aus und wurde in der Folge abgeändert. Vgl. Botschaft ZPO, z. B. Punkt 6.2, Auswirkungen auf die Kantone: «Das Prinzip der Kostenneutralität ist ein Leitgedanke des Entwurfs», S. 7410; auch: «Hauptanliegen der Kantone: Keine Mehrkosten!», S. 7238 und: «Die Kantone bestimmen den Preis der Justiz», S. 7244

6 Vergleiche zum Beispiel Meier, S. 421 f.; Marti, S. 21 ff.; Meier/Schindler, S. 80; Zotsang, S. 118 f., S. 246 f.; im Gegensatz dazu sieht STÄHELIN 2017b, Rz. 24 ff., aber keinen Änderungsbedarf bezüglich der Kostenvorschusspflicht und spricht sich nur für eine Anpassung der Liquidationsregelung aus.

7 Vergleiche zum Beispiel SRF, Sendung ECO vom 23.1.2018, JeAn FrançoIs TANDA, Unbezahlbare Gerechtigkeit, Gerichtskosten für KMU zu hoch, und div. Artikel in der NZZ mit allgemeiner Kritik an der neuen Prozesskostenregelung: u. a. NZZ vom 12.12.2016, Katharina Fontana, Hoher Preis für Justitia; NZZ vom 12.4.2017, Brigitte Hürlimann und Lucien Scherer, Die Schlitzohren profitieren; NZZ vom 13.2.2018, Daniel Gerny, Der Gang vor Gericht wird zum Luxusgut - im Extremfall droht Klägern der Ruin.

8 Vorgeschlagen wurden die Reduzierung des Kostenvorschusses auf $50 \%$ und der Verzicht auf die Liquidationsregelung, vgl. Vernehmlassungsvorlage und Erläuternder Bericht Rev. ZPO, S. 15 f. In der Vernehmlassung wiederum ablehnend die Rückmeldungen der meisten Kantone (aus Kostengründen), ebenso ablehnend die grösseren Wirtschaftsverbände, zustimmend dagegen Gewerbeverbände, Mitte-Links-Parteien, Anwaltsverbände und Vertreter der juristischen Lehre, vgl. Ergebnisbericht Rev. ZPO, S. 8 ff. 
habe allerdings seit Einführung der ZPO der Vergleichsdruck stark zugenommen. Dies könne durchaus als indirekte Auswirkung der neuen Kostenregelung der ZPO betrachtet werden. Da beide Parteien wüssten, dass ein Gang vor Gericht aus Kostengründen de facto nicht in Frage komme, sei eine Einigung zwingend. Dies setze vor allem eine wirtschaftlich schwächere Partei unter Druck und verstärke das Machtgefälle (vergleiche auch Staenelin 2017a S. 176 ff., S. 188 f. und Sтоцz, S. 21 f.). Ein Prozess mit einem neutralen Richter, einer neutralen Richterin wäre in einigen Fällen vorteilhafter und könnte das Machtgefälle senken, da aus der richterlichen Fragepflicht eine Hinwirkung zur Unterstützung der (schwächeren) Parteien abgeleitet werden kann.

[26] Insgesamt vermitteln die aufgeführten Argumente ein klares Bild und bestätigen einen Befund aus dem vorangegangenen Kapitel: Die ZPO baut mit den Kostenregelungen eine Schranke auf, die KMU aufgrund fehlender Ressourcen oftmals nicht zu bewältigen vermögen. Es liegt ein faktisches Vollzugsdefizit vor, allerdings nicht wegen fehlender Ressourcen der Vollzugsinstanzen, sondern der durch den Vollzug betroffenen Rechtssuchenden, den KMU.

\subsection{Justiz enttäuscht Gerechtigkeitserwartungen}

[27] Richterinnen und Richter stehen unter hohem Druck, Verfahren mit einem Vergleich abzuschliessen (vgl. dazu etwa die grundlegende Untersuchung von EgLi 1995/1996 sowie die neuere Untersuchung von Schweizer 2018, Rz. 1 ff./Rz. 6). Eine hohe Vergleichsquote gilt als Zeichen für Effizienz und Zufriedenheit. Bis zu 90 Prozent der Verfahren werden gemäss Schätzungen der von uns Befragten mit einem Vergleich abgeschlossen (vgl. auch Schweizer 2018; Platz, S. 1 f.), wobei dies in erster Linie prozessökonomischen Gründen geschuldet ist. Die Vorteile des Vergleichs liegen darin, dass auf zeitintensive und teure Beweiserhebungen verzichtet werden kann, kreative Lösungen über die Anträge hinaus gefunden werden und ein zeitintensiver und teurer Weiterzug an die nächste Instanz entfällt.

[28] Dieser hohe Anteil an Vergleichen kann aber bei KMU und Mikrounternehmen zu einer Enttäuschung der Gerechtigkeitserwartung in die Gerichte führen, was sich wie folgt begründen lässt:

- Zunächst wird in der Literatur kritisiert, dass das verfassungsmässige Recht auf Zugang zu einem Gericht gemäss Artikel 29a BV nicht auf einen Zugang zu einer Vergleichsverhandlung verkürzt werden sollte (Meier 2010, S. 428). Wird dieser Trend zu Vergleichsverhandlungen von den Gerichten gefördert, kann dies die Gerechtigkeitserwartung der KMU trüben.

- Wichtiger ist allerdings ein zweiter Aspekt: Sind die eigenen Strategien der KMU zur Konfliktlösung nicht erfolgreich und wird die Justiz als «Pannenhilfe» (Stolz 2000, S. 17) dennoch in Anspruch genommen, sind sowohl Mikro- wie auch Mittelunternehmen davon überzeugt, dass sie Recht haben und dass sie dieses auch bekommen werden (KaufmanN et al., S. 134): Ab einem gewissen Punkt der Konflikteskalation will ein Unternehmer nicht mehr in einen erneuten Kompromissversuch einwilligen. Vielmehr «will er es jetzt wissen» (Kaufmann et al. 2010, S. 134, S. 140 ff.): Das Gericht soll Gerechtigkeit schaffen. Wird der Unternehmer nun aber mit einem Vergleich «abgespiesen», führt dies zu Frustration. Das Unternehmen fühlt sich betrogen mit einem Ergebnis, das es durch Nachgeben und $\mathrm{Ku}-$ lanz ebenso hätte erzielen können, allerdings ohne Zeit- und Geldverlust (KAufmann et al. 2010, S. 138 f.; vgl. auch Girsberger 2003, S. 645). Die fiktionale Gerechtigkeitserwartung 
der Mikrounternehmen wird durch den Abschluss eines Vergleichs enttäuscht. Man könnte nun einwenden, dass es den KMU freistehen würde, einen Vergleich auszuschlagen und auf einen Prozess mit einem Entscheid zu bestehen. In der Praxis dürfte es aber vielfach so sein, dass die KMU aufgrund ihrer weit geringeren fachlichen Kompetenzen und aufgrund ihrer beschränkten Ressourcen sich contre cœur einem Vergleich anschliessen.

[29] Die enttäuschte Gerechtigkeitserwartung ist in diesem Fall denn auch nichts anderes als das Ausbleiben der Wirksamkeit der gerichtlichen Streitschlichtung. Ist aber keine Wirksamkeit zu erwarten und spricht sich dies herum, werden Kosten und Mühen für die Beschreitung des Rechtswegs nicht in Kauf genommen.

\subsection{Informationsdefizite der KMU sind hoch}

[30] Es lassen sich theoretische wie empirische Befunde anfügen, die nahelegen, dass fehlende Information die Distanz zwischen KMU und Gerichten erhöht und mit einer verminderten Anrufung der Gerichte einhergeht. Diese Annahme ist plausibel, ist doch das Fehlen von Informationen und Beratung oftmals ein wesentlicher Grund dafür, dass öffentliche Massnahmen die Zielgruppen nicht erreichen und daher keine Wirkungen zu entfalten vermögen (RIEDER et al. 2014: S. 575-576). Betrachten wir diesen Aspekt zunächst aus einer theoretischen Perspektive heraus.

[31] Rehbinder bemerkte bereits 1972, dass die Funktion der Verhaltenssteuerung in der Gesellschaft mangels Kenntnis des Rechts auf andere soziale Ordnungssysteme wie Regeln der Sitte, der Moral oder der Konvention übergehe (Rehbinder Reprint 1996, S. 26). Geht man weiter davon aus, dass KMU die im Recht verankerten Rollenerwartungen nicht kennen, liegt der Schluss nahe, dass KMU sich auf das «sozial Übliche» stützen. Dieses wird zum Surrogat für die Institution Recht als Ordnungsinstrument unternehmerischen Handelns (Von Escher 2008, S. 64 f.). Die nachfolgend beschriebene zurückhaltende Information und Kommunikation der Gerichte würde diesen Effekt noch verstärken und zu einer verstärkten Nutzung interner Institutionen führen.

[32] Es lassen sich aber auch empirische Befunde finden, die fehlende Informationen als Grund für den seltenen Gang von KMU an Zivilgerichte belegen. Gerichte kommunizieren primär und traditionell über ihre Urteile (SAXer/SAxer 2015, S. 4 ff.). Diese sind aber in einer abstrakten Fachsprache verfasst und bedürfen der Übersetzung. Gründe dafür sind einerseits die facettenreiche Materie «Recht», andererseits die Eigentümlichkeiten der Juristensprache und die spezifische Gerichts- und Prozessterminologie (Santschi Kallay 2017/2018, S. 17). Davon ausgehend sprechen andere Autoren sogar von einer eigentlichen Sprach- und Wissensbarriere (GоsтомzYк 2006, S. 146 ff.). Diese abzubauen ist üblicherweise Aufgabe der Gerichtsberichterstattung in den Medien. Die Voraussetzungen dafür wären an sich durchaus gegeben: Gemäss einer Umfrage interessieren sich über drei Viertel der Befragten in der Schweiz für Medienberichte über Gerichtsprozesse (Rieder/Schwenkel 2014, N 26). Allerdings fühlen sich 59 Prozent schlecht oder eher schlecht über die Gerichte in ihrem Kanton informiert (SCHwenkel 2016, N 231 f.). Damit ist eine Diskrepanz zwischen dem Interesse der Bevölkerung an der Rechtsprechung und den dazu erhältlichen Informationen zu konstatieren. Wie Gerichte funktionieren, wie Verfahren ablaufen und was Richterinnen und Richter konkret tun, ist demnach grossen Teilen der Bevölkerung unbekannt. Dieser Befund dürfte sich auch auf KMU übertragen lassen - und noch viel mehr auf die vom Firmeninhaber oder von der Firmeninhaberin geprägten Mikrounternehmen. 
[33] Es liegt nahe, aufgrund dieser Befunde mehr Information und Kommunikation von Seiten der Gerichte zu fordern: Eine kontinuierliche, eigenständige und aktive gerichtliche Informationstätigkeit entspricht gemäss verschiedenen Autorinnen und Autoren denn auch einer modernen Justiz und könnte helfen, das Wissen über die Justiz und die Nutzung der Rechtsmittel zu erhöhen (Santschi Kallay 2018, S. 16; Metz 2018 oder auch z.G. Richterzeitung 2018/2). Wie weit die Gerichte diese Befunde und Anregungen aufnehmen, ist offen. Gegenwärtig dürfte aber gelten, dass die geringe Anrufung der Zivilgerichte zur Streitschlichtung durch KMU und Mikrounternehmen durch unzureichende Information mitverursacht wird.

\subsection{Zwischenfazit: Hohe Kosten, enttäuschte Erwartungen und fehlende Informationen hemmen die Anwendung und Wirksamkeit zivilprozessualer Streitschlichtungsinstrumente}

[34] Fassen wir die Signale zusammen, welche die Justiz in Richtung KMU aussendet, so werden die Ergebnisse aus Ziffer 2 bestätigt.

- Die Prozesskosten dürften die KMU und insbesondere die Mikrounternehmen von einem Gang an die Gerichte abhalten. Gerade bei Mikrounternehmen sind die zu leistenden Vorschüsse nicht aufzubringen, oder die Prozesskostenfolgen könnten das Unternehmen in seiner Existenz gefährden. Es liegt ein faktisches Vollzugsdefizit vor: Die Ressourcen auf Seiten der KMU und Mikrounternehmen fehlen, um das Recht anzuwenden.

- Geht ein KMU oder Mikrounternehmen dennoch einmal den Weg ans Gericht, droht es bei einem Vergleich zu enden, obwohl es einen richterlichen Entscheid angestrebt hat. Die Gerechtigkeitserwartung wird enttäuscht, namentlich weil der Vergleich auch ohne Gerichte und mit tieferen Kosten erreichbar gewesen wäre. Die enttäuschte Gerechtigkeitserwartung entspricht dabei aus Sicht der KMU einer fehlenden Wirksamkeit zivilprozessualer Streitschlichtung.

- Schliesslich ist die heutige Information bei der Bevölkerung über die Gerichte eher als tief zu bezeichnen. Dies dürfte auch für die KMU gelten. Solange die Gerichte nicht stärker informieren, wird bei einem Teil der KMU fehlendes Wissen mitverantwortlich sein, dass sie eher auf interne Regeln und Verfahren zur Konfliktlösung setzen als auf den Gang vor ein Zivilgericht. Dies reduziert die Wirksamkeit im Sinne der Gerechtigkeitserwartung und des Entscheids über Recht und Unrecht zusätzlich.

\section{Schlussfolgerungen}

[35] Wir fassen die zentralen Punkte zusammen, die heute das Verhältnis zwischen KMU und Justiz prägen und aus unserer Sicht für die geringe Anwendung und Wirksamkeit zivilprozessualer Streitschlichtungsinstrumente bei KMU und Mikrounternehmen verantwortlich sind. Anschliessend diskutieren wir, ob und wie weit ein Veränderungsbedarf besteht. 


\subsection{Endogene und exogene Gründe für die geringe Nutzung von Zivilgerichten durch KMU}

[36] Gesamthaft betrachtet können wir heute von einem Nicht-Verhältnis zwischen KMU und Justiz sprechen: Zwar besteht mit den Zivilgerichten eine Möglichkeit zur Streitschlichtung für die KMU. Fehlendes Wissen und Ressourcen auf Seiten der KMU, effektivere interne Schlichtungsmechanismen, hohe Kosten von Zivilprozessen, enttäuschte Gerechtigkeitserwartungen und zurückhaltende Informationsaktivitäten der Gerichte verhindern aber, dass KMU die Zivilgerichte überhaupt in Anspruch nehmen.

[37] Aus der Vollzugsforschung wissen wir, dass das Zusammenspiel der an der Umsetzung einer Politik beteiligten Akteure, deren Ressourcenausstattung und Informationsaktivitäten die Wirksamkeit einer Politik wesentlich beeinflussen, wenn nicht gar bestimmen (RIEDER et al. 2014: S. 584-585). Übertragen wir diese Überlegungen auf die Nutzung von Zivilgerichten durch KMU, zeigen sich Analogien: Beim heutigen Informationsstand der KMU über Verfahren und Funktionsweise der Gerichte, bei den bestehenden Prozesskosten sowie bei der aktuellen Art und Weise der Kommunikation der Gerichte wäre es eine Überraschung, wenn KMU die Zivilgerichte häufiger anrufen würden. Entsprechend ist davon auszugehen, dass die Wirkung der entsprechenden Rechtsprechung klein sein dürfte. Dies dürfte auch dann gültig sein, wenn Urteile ergehen, die materiell für die KMU bedeutsam sind. Solange diese aber nicht verständlich kommuniziert und von den KMU rezipiert werden, dürfte die Wirksamkeit der Urteile aber klein sein.

\subsection{Veränderungsbedarf: Pro und Contra}

[38] Es stellt sich nun die Frage, ob der obige Befund einfach hingenommen oder ob eine Veränderung angestrebt werden soll? Für Letzteres lassen sich Gründe dagegen und dafür finden.

[39] Für einen Verzicht auf eine Veränderung spricht die geringe Problematisierung der Thematik durch Unternehmen, Verbände und Politik. Weiter scheint die offenbar seit Jahrzehnten bestehende geringe Nutzung der Justiz durch KMU ein Indiz dafür zu sein, dass sich die Unternehmen mit der Situation abgefunden oder zumindest arrangiert haben. Es scheint politisch akzeptiert zu sein, dass die Kostenfolgen für die Kantone, welche die Ausgaben für die Gerichte tragen müssen, einen höheren Stellenwert haben als der Zugang der KMU zu den Gerichten und die Stärkung des Rechtsschutzes. ${ }^{9}$ Und schliesslich kann die geringe Informationstätigkeit der Gerichte durchaus auch in positivem Lichte gesehen werden: Die Nicht-Information kann zu einer stabilen Fiktion des Vertrauens in die Justiz führen. Solange diese hoch ist, wäre somit kein Handlungsbedarf gegeben.

[40] Was würde für eine Veränderung der heutigen Situation sprechen? Folgen wir der Argumentation, wonach Information und Kommunikation den Austausch fördert und die Anwendung des Rechts unterstützt, so wäre in der Konsequenz eine verstärkte Kommunikationspolitik der Justiz anzumahnen. Die aktuell zurückhaltende Information der Gerichte über deren Regeln und Mechanismen wäre somit zu überdenken. Gerichte könnten beispielsweise über die Vorteile der Vergleiche informieren und die Möglichkeiten und Grenzen des Rechts aufzeigen. Mit der NichtKommunikation wird eine falsche Fiktion genährt, die auf Dauer nicht aufrechtzuerhalten ist.

9 Vergleiche dazu Erläuternder Bericht Rev. ZPO, S. 15 f.; Ergebnisbericht Rev. ZPO, S. 9 f; vgl. auch mahnend der Bundesrat in Botschaft Revision ZPO, S. $2712 \mathrm{ff}$. 
Daher wären auch neue Wege zu suchen, Entscheide und Gerichtsverfahren in verständlicher Sprache zu erläutern. Die Kosten der Verfahren wären zu reduzieren. Auf diese Weise könnten die Inanspruchnahme zivilprozessualer Streitschlichtung durch KMU sowie die Wirksamkeit der Rechtsprechung erhöht werden.

[41] Welche Argumente wiegen schwerer? Momentan lässt sich dies schwer oder (noch) nicht abschliessend entscheiden. Trotz der vorgetragenen theoretischen wie empirischen Argumente sind die Grundlagen dürftig: Zwar wird an den Gerichten seit jeher eine Vielzahl von Daten erhoben. Leider lassen diese Daten aber vielfach keine Rückschlüsse auf die hier diskutierte Problematik zu. So sind keine gesicherten Zahlen zu der Anzahl von Klagen von KMU und Mikrounternehmen erhältlich, und wir wissen nicht, welchen Einfluss die Einführung der ZPO auf die Anzahl der seitens KMU eingereichten Klagen gehabt hat. Angesichts der Bedeutung der KMU für die Wirtschaft und die Gesellschaft ist es aus unserer Sicht im öffentlichen Interesse, hier verbesserte Grundlagen zu schaffen, damit die in diesem Beitrag formulierten Zusammenhänge überprüft werden könnte. So liesse sich denn auch die Frage nach einem Veränderungsbedarf besser beantworten.

[42] Für den Bundesrat wiegen die Argumente für eine Veränderung des Status quo offenbar schwerer. So hat er in seiner Botschaft zur Revision der Zivilprozessordnung dem Parlament eine Senkung der Kostenvorschüsse und eine Verbesserung der Statistik im Bereich der ZPO vorgeschlagen (Botschaft Revision ZPO, S. 2712 ff. / S. 2777 f.). Ob und wie weit das Parlament diesem Vorschlag folgen wird, bleibt abzuwarten.

Seraina Herzberg, MLaw, seraina.herzberg@zuerich.ch.

Stefan Rieder, Dr. rer. pol., Geschäftsführer INTERFACE Politikstudien Forschung Beratung sowie ständiger Lehrbeauftragter an der Universität Luzern, rieder@interface-pol.ch.

\section{Materialien und Literaturverzeichnis}

Botschaft zur Schweizerischen Zivilprozessordnung vom 28. Juni 2006, BB1 20067221 ff. (zit. Botschaft ZPO).

Botschaft zur Änderung der Schweizerischen Zivilprozessordnung (Verbesserung der Praxistauglichkeit und der Rechtsdurchsetzung) vom 26. Februar 2020, BBl 20202697 ff. (zit. Botschaft Revision ZPO).

EgLI, URs (1995/1996): Vergleichsdruck im Zivilprozess, Diss. Zürich/Berlin 1996.

Erläuternder Bericht zur Änderung der Zivilprozessordnung (Verbesserung der Praxistauglichkeit und der Rechtsdurchsetzung) vom 2. März 2018 (zit. Erläuternder Bericht Rev. ZPO).

Girsberger, Daniel (2003): Eine optimale Streiterledigung für KMU?, in: Forstmoser Peter / Crone Hans Caspar (Hrsg.): Neuere Tendenzen im Gesellschaftsrecht, FS für Peter Forstmoser zum 60. Geburtstag, 639 ff., Zürich.

Gostomzyк, Tовias (2006): Die Öffentlichkeitsverantwortung der Gerichte in der Mediengesellschaft, Baden-Baden.

Kaufmann, Ronald/von Escher, Hadumoth/Furrer, Andreas/Girsberger, Daniel (2010): KMU und Konflikte - Blick auf die Wirklichkeit in Schweizer Unternehmen, Bern.

Kiwit, Daniel/Voigt, Stefan (1997): Überlegungen zum institutionellen Wandel unter Berücksichtigung des Verhältnisses interner und externer Institutionen, in: ORDO - Jahrbuch für die Ordnung von Wirtschaft und Gesellschaft, Band 46, Berlin, $117 \mathrm{ff}$ 
Luhmann, Niklas (2014): Vertrauen, Ein Mechanismus der Reduktion sozialer Komplexität, 5. Aufl., Konstanz.

Marti, Arnold (2017): Die Kosten im heutigen Zivilprozess - was bleibt vom Grundsatz der wohlfeilen Rechtspflege, in: «Justice - Justiz - Giustizia» 2017/3 vom 7. September 2017, Bern.

MeIer, IsaAK (2010): Schweizerisches Zivilprozessrecht - eine kritische Darstellung aus der Sicht von Praxis und Lehre, Zürich.

MeIER, IsAaK/Schindler, Riccarda (2015): Unerschwinglichkeit der Rechtsdurchsetzung - eine Verweigerung des Zugangs zum Gericht? In: Fellmann Walter / Weber Stephan (Hrsg.), Haftpflichtprozess 2015, Tagungsband, 29 ff.

Metz, Markus (2018): Kommunikation der Gerichte, in: «Justice - Justiz - Giustizia» 2018/2 vom 14. Juni 2018, Bern.

Platz, Ernst (2014): Der Vergleich im schweizerischen Recht, Diss. St. Gallen.

Rabbel, Christian (2007): Beratungsresistenz in kleineren und mittleren Unternehmen, Saarbrücken.

Rehbinder, Manfred (1972, Reprint 1996): Rechtskenntnis, Rechtsbewusstsein und Rechtsethos als Problem der Rechtspolitik, in: Jahrbuch für Rechtssoziologie und Rechtstheorie, Band 3, Bad Feilnbach, S. 25 ff.

«Justice - Justiz - Giustizia» 2018/2 vom 14. Juni 2018, Schwerpunkt-Ausgabe: Gerichtskommunikation, Bern (zit. Richterzeitung 2018/2).

Rieder, Stefan/Schwenkel, Christof (2014): Die Wahrnehmung der Justiz durch die Bevölkerung, Resultate einer Bevölkerungsbefragung in 26 Kantonen, in: «Justice - Justiz - Giustizia» 2014/1 vom 6. März 2014, Bern.

Rieder, Stefan/Balthasar, Andreas/Kissling-Naef, Ingrid (2014): Vollzug und Wirkung öffentlicher Politik, in: Knoepfel, P., Papadopoulos, Y., Sciarini, P., Vatter, A., Häusermann, S. (Hrsg.): Handbuch der Schweizer Politik = Manuel de la politique suisse, S. 563-598.

Santschi Kallay, Mascha (2017/2018): Externe Kommunikation der Gerichte, rechtliche und praktische Aspekte der aktiven und reaktiven Medienarbeit der Judikative, Diss. Zürich/Bern.

SAXer, Urs (2015): Die Justiz als verkannte Staatsgewalt? In: Saxer Urs (Hrsg.), Kommunikation der Gerichte, Recht und Praxis, Zürich.

Schmid, Gian Andrea (2017): Zivilprozess als «Rechtswegbarriere», in: plädoyer 6/2017, Zürich, 12 f.

Schweizer, Mark (2018): Praxis der Vergleichsverhandlung, in: «Justice - Justiz - Giustizia» 2018/4 vom 13. Dezember 2018, Bern.

Schwenkel, Christof (2016): Der Einfluss kantonaler Justizsysteme auf das Vertrauen der Bevölkerung in die Gerichte, Bern.

StÄhelin, Beda Andreas (2017): Rechtsverfolgungskosten und unentgeltliche Rechtspflege im Lichte der Rechtsgleichheit, Diss. Zürich (zit. StÄHELIN 2017a).

StÄhelin, Beda Andreas (2017): Gerichtskostenvorschusspflicht und Zugang zum Recht, in: «Justice - Justiz - Giustizia» 2017/3 vom 7. September 2017, Bern (zit. STÄHELIN 2017b).

Stolz, Peter (2000): Mittelständische Unternehmungen (KMU) und Justiz - eine ökonomische Analyse, Zürich.

Übersicht über das Ergebnis des Vernehmlassungsverfahrens, Revision der Zivilprozessordnung (Verbesserung der Praxistauglichkeit und der Rechtsdurchsetzung) vom 29. Januar 2020 (zit. Ergebnisbericht Rev. ZPO).

Voigt, Stefan (2009): Institutionenökonomik, 2. Aufl., Paderborn.

von Escher, HАDUmoth (2008): KMU-Beratung durch Rechtsanwälte, Eine rechtstatsächliche Untersuchung, Diss. Luzern.

Zotsang, Dheden C. (2015): Prozesskosten nach der Schweizerischen Zivilprozessordnung, Diss. Zürich. 